\title{
EXTRAÇÃO DE MINERAIS PELO CAPIM MARANDU FERTILIZADO COM ESTERCO DE GALINHA SEM E COM ESCARIFICAÇÃO
}

\author{
Edson Sadayuki Eguchi ${ }^{1}$, Ulysses Cecato ${ }^{2}$, Antonio Saraiva Muniz ${ }^{3}$, Samuel Laudelino da Silva ${ }^{4}$, \\ Cristiano da $\mathrm{Cruz}^{5}$
}

\begin{abstract}
RESUMO - O uso de esterco de aves como fonte de nutrientes para pastagens em áreas degradadas é uma forma de destinação ambientalmente correta para reduzir os impactos ambientais. O objetivo do trabalho foi avaliar ao final de um ano os minerais presentes e sua extração pelo capim Marandu fertilizado com esterco de galinha sem e com uso de arado escarificador no solo. O delineamento utilizado foi em blocos ao acaso com quatro repetições num arranjo fatorial $5 \times 2$, com cinco doses $\left(0 ; 1,037 ; 2,074 ; 4,148 ; 6,222 \mathrm{Mg} \mathrm{ha}^{-1}\right)$ de esterco, sem e com manejo de escarificação do solo e mais um tratamento adicional com adubação mineral NPK contidos em 2,074 Mg ha ${ }^{-1}$. Os cortes foram efetuados conforme interceptação luminosa de $95 \%$ do dossel na altura de $0,15 \mathrm{~m}$ de resíduo. $\mathrm{Na}$ interação dose de esterco x manejo foi significativa para $\mathrm{Ca}, \mathrm{Mg}, \mathrm{N}, \mathrm{Cu}$ e Zn, efeito de dose para $\mathrm{P}$ e sem efeito significativo para o K. Na extração anual a interação dose de esterco x manejo com escarificação houve ajuste linear, o N é o mineral de maior extração, seguido pelo $\mathrm{K}, \mathrm{Ca}, \mathrm{Mg}, \mathrm{P}$ e Zn. $\mathrm{Na}$ extração mineral por corte efetuado com interação dose sem escarificação houve ajuste ao modelo linear decrescente entre as doses para o Ca, relacionado ao efeito da diluição em razão dos números de cortes. As fertilizações com esterco de galinha e manejo do solo alteram a extração anual dos principais minerais relacionados.
\end{abstract}

Palavras-chave: braquiária, extração, fertilização, manejo, química.

\section{CORRETO: MINERALS EXTRACTION BY MARANDU GRASS FERTILIZED WITH CHICKEN MANURE WITH OR WITHOUT SOIL CHISELING}

\begin{abstract}
The use of poultry manure as a source of nutrients for pasture in degraded areas is an environmentally sound form of disposal to reduce environmental impacts. The objective of this work was to evaluate at the end of one year the minerals present and their extraction by the Marandu grass fertilized with chicken manure without and with the use of chiseling plowing in the soil. The experimental design was a randomized block design with four replications in a $5 \times 2$ factorial arrangement, with five doses $(0 ; 1.037 ; 2.074 ; 4.148 ; 6.222$ $\mathrm{Mg} \mathrm{ha}^{-1}$ ) of manure, without and with soil chiseling management. And, an additional treatment with NPK mineral fertilization contained in $2.074 \mathrm{Mg} \mathrm{ha}^{-1}$. The cuts were done according to light interception of $95 \%$ of the canopy at a height of $0.15 \mathrm{~m}$ of residue. In the interaction between dose of manure and management, it was significant for Ca, Mg, $\mathrm{N}$, Cu and $\mathrm{Zn}$, dose effect for $\mathrm{P}$ and no significant effect for $\mathrm{K}$. $\mathrm{N}$ is the mineral with the highest extraction, followed by $\mathrm{K}, \mathrm{Ca}, \mathrm{Mg}, \mathrm{P}$ and $\mathrm{Zn}$. In the mineral extraction per cut performed with dose interaction without chiseling, there was adjustment to the linear model decreasing between the doses for Ca related to the effect of the dilution due to the number of cuts. Fertilization with chicken manure and soil management significantly altered the annual extraction of the main related minerals.
\end{abstract}

Keywords: chemical, extraction, fertilization, management, palisadegrass.

\footnotetext{
${ }^{1}$ Professor do Departamento de Zootecnia, Universidade do Estado de Mato Grosso - UNEMAT. Caixa Postal No 181 CEP 78250-000 Pontes e Lacerda (MT).E-mail: eguchi@unemat.br

${ }^{2}$ Professor do Departamento de Zootecnia, Universidade Estadual de Maringá - UEM. CEP 87020-900 Maringá (PR). Email: ucecato@uem.br

${ }^{3}$ Professor do Departamento de Agronomia, Universidade Estadual de Maringá - UEM. CEP 87020-900 Maringá (PR). Email: asmuniz@uem.br

${ }^{4}$ Professor do Departamento de Zootecnia, Universidade do Estado de Mato Grosso - UNEMAT. Caixa Postal No 181 CEP 78250-000 Pontes e Lacerda (MT). E-mail: samuel@unemat.br

${ }^{5}$ Professor do Departamento de Zootecnia, Universidade do Estado de Mato Grosso - UNEMAT. Caixa Postal No 181 CEP 78250-000 Pontes e Lacerda (MT).E-mail: cristianodacruz@unemat.br
} 


\section{INTRODUÇÃO}

Restabelecer os níveis adequados de disponibilidade de nutrientes, por meio de correção e fertilização é a forma mais eficiente de recuperação dos processos produtivos das pastagens. Segundo Ashjaei et al. (2011), o esterco de galinha é um importante fertilizante orgânico. Além da sua composição química e sua relação $\mathrm{C} / \mathrm{N}$, a mineralização é contínua ao longo do tempo com a liberação de $\mathrm{NH}_{4}{ }^{+} \mathrm{e}$ consequente formação de nitrato no solo (Azeez \& Averbeke, 2010).

Segundo o Ministério da Agricultura Pecuária e Abastecimento (Brasil, 2009), é permitido o uso da cama ou esterco de aves em pastagens e capineiras, com 40 dias de carência após processo de fertilização e incorporação. Sem definir o processo de incorporação mecânica, o uso racional deste resíduo orgânico se faz necessário com estudos sobre o potencial aumento na produção do capim Marandu em áreas degradadas.

A escarificação do solo rompe camadas compactadas na superfície, reduz a densidade e aumenta a macroporosidade e porosidade total, favorece a aeração e ação de microrganismos sobre o esterco aplicado (Eguchi et al., 2016). A fertilidade do solo e as estratégias de manejo podem resultar em variações na estrutura do dossel no momento do pastejo ou corte com influência no desempenho animal (Pedreira et al., 2009) em decorrência dos seus efeitos nos teores minerais e no valor nutritivo da forragem ofertada e consumida (Trindade et al., 2007; Difante et al., 2009).

O estudo na concentração e extração mineral pelo capim Marandu (Brachiaria brizantha (Hochst ex. A. Rich.) Stapf cv. Marandu), sob doses e fontes de nitrogênio com intervalos fixos entre cortes, foram realizados por Primavesi et al. (2006) e Costa et al. (2009ab). Segundo Pedreira et al. (2009), a melhor estratégia de desfolhação é de $95 \%$ de interceptação luminosa com altura de resíduo de $0,15 \mathrm{~m}$ para o acúmulo de folhas. Para Voltolini et al. (2010), com esse manejo, possibilita aplicar intervalos variados e menores de pastejo com efeitos positivos na produção e no valor nutritivo da planta forrageira.

Dentro deste contexto, objetivou-se avaliar os minerais presentes e extraídos pela massa produzida com $95 \%$ de interceptação luminosa pelo capim Marandu fertilizado com esterco de galinha e manejado sem e com escarificação do solo.

\section{MATERIAL E MÉTODOS}

O experimento foi conduzido na Fazenda Experimental de Iguatemi ( $23^{\circ} 25^{\prime} \mathrm{S}$ e $51^{\circ} 57^{\prime} \mathrm{W}$ a $550 \mathrm{~m}$ de altitude), da Universidade Estadual de Maringá (UEM), Maringá - PR. Com 10 anos de pastejo, o capim Marandu foi estabelecido em Latossolo Vermelho distrófico com $87,25 \%$ de areia na camada $0-0,2 \mathrm{~m}$. Na área experimental a análise química do solo coletado a $0-0,2 \mathrm{~m}$, apresentou entre bloco os teores médios de $\mathrm{pH}\left(\mathrm{H}_{2} \mathrm{O}\right)=6,00 ; \mathrm{C}=8,23$ $\mathrm{g} \mathrm{dm}^{-3} ; \mathrm{P}=6,28 \mathrm{mg} \mathrm{dm}{ }^{-3} ; \mathrm{H}^{+}+\mathrm{Al}^{+3}=2,54 ; \mathrm{Ca}^{+2}=0,87$; $\mathrm{Mg}^{+2}=0,57 ; \mathrm{K}^{+}=0,13 \mathrm{cmol}_{\mathrm{c}} \mathrm{dm}^{-3} \mathrm{e} \mathrm{Fe}=180,86 ; \mathrm{Zn}=4,04$;

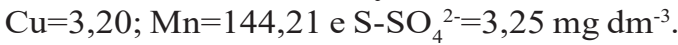

O esterco de galinha da linhagem Hy Line W36 em postura com gaiolas, foi armazenado à sombra por 45 dias e coberta com lona. A análise química do esterco apresentou $60 \%$ de matéria orgânica, $\mathrm{N}_{\text {total }}=6,64 \%$; $\mathrm{CaO}=3,53 \% ; \mathrm{MgO}=5,51 \% ; \mathrm{K}_{2} \mathrm{O}=3,73 \% ; \mathrm{P} 2 \mathrm{O} 5=$ $2,41 \% ; \mathrm{C} / \mathrm{N}=4,96: 1 ; \mathrm{Cu}=220,10 \mathrm{mg} \mathrm{kg}^{-1} ; \mathrm{Mn}=1226,90$ $\mathrm{mg} \mathrm{kg}{ }^{-1} ; \mathrm{Zn}=368,00 \mathrm{mg} \mathrm{kg}^{-1}$ e $\mathrm{pH}\left(\mathrm{H}_{2} \mathrm{O}\right)=6,98$.

Para elevação da saturação de bases do solo em $50 \%$ fez a aplicação manual de $490 \mathrm{~kg} \mathrm{ha}^{-1}$ de calcário dolomítico (32\% CaO e 15\% MgO) sobre o capim Marandu no mês de agosto de 2012 e depois de 25 dias corte de uniformização com roçadora a $0,10 \mathrm{~m}$ do solo.

A dose de $50 \mathrm{~kg} \mathrm{ha}^{-1}$ de $\mathrm{P}_{2} \mathrm{O}_{5}$ foi baseada na manutenção de espécies do grupo III (Menezes et al., 2004; Oliveira, 2003) presente em 2,074 $\mathrm{Mg} \mathrm{ha}^{-1}$ de esterco. O delineamento experimental foi em blocos ao acaso com quatro repetições num arranjo fatorial $5 \times 2$, cinco doses $\left(0,1,037,2,074,4,148,6,222 \mathrm{Mg} \mathrm{ha}^{-1}\right)$ de esterco de galinha e dois manejos sem e com escarificação do solo. Foi acrescentado um tratamento com adubação mineral em NPK contido em 2,074 $\mathrm{Mg} \mathrm{ha}^{-1}$ de esterco de galinha com $138 \mathrm{~kg} \mathrm{ha}^{-1}\left(\mathrm{~N}_{\text {total }}\right.$-Nitrato de Amônio), $50 \mathrm{~kg} \mathrm{ha}^{-1}\left(\mathrm{P}_{2} \mathrm{O}_{5}-\right.$ Superfosfato triplo), $77 \mathrm{~kg} \mathrm{ha}^{-1}\left(\mathrm{~K}_{2} \mathrm{O}-\right.$ Cloreto de Potássio) para efeito de comparação de médias.

No mês de setembro foram aplicados na área experimental $270 \mathrm{~kg} \mathrm{ha}^{-1}$ de gesso agrícola e em cada parcela correspondente $(6 \times 4) \mathrm{m}$ dose única de esterco. No tratamento com adubação mineral uma dose única de $\mathrm{P}_{2} \mathrm{O}_{5}$ e $1 / 3$ da dose para $\mathrm{N}_{\text {total }}$ e $\mathrm{K}_{2} \mathrm{O}$ parcelados a cada 60 dias. E, posterior escarificação com Arado Descompactador Tanden da marca IKEDA (DPT320M) que apresentava disco de corte posicionado anteriormente a cada haste helicoidal com ponteira inclinada de laminas de aço, a $0,2 \mathrm{~m}$ de profundidade. 


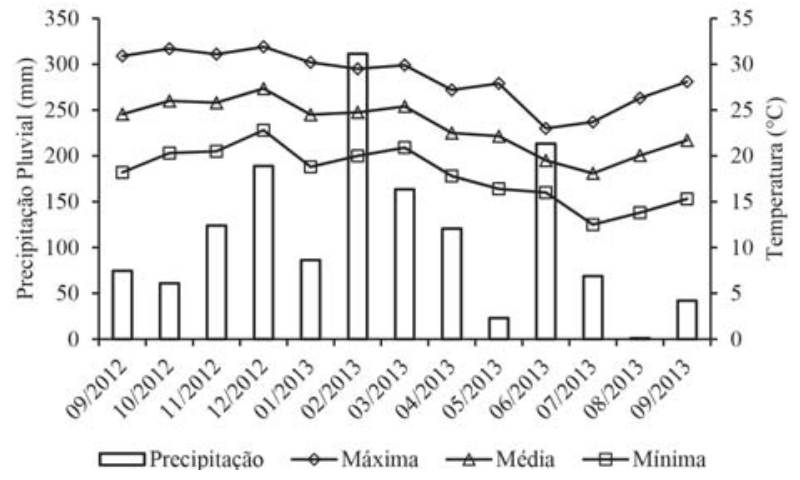

Figura 1 - Precipitação pluvial e temperaturas, no período experimental de setembro de 2012 a 2013. Fonte: Eguchi et al. (2016).

O manejo do capim Marandu foi baseado na interceptação luminosa (IL) de 95\% do dossel forrageiro medido com aparelho AccuPAR modelo LP-80 PAR/ LAI Ceptometer com leituras semanais e rebaixado com aparador mecânico a altura de resíduo de 0,15 m e remoção de toda a massa cortada. Um quadrado de ferro com $0,25 \mathrm{~m}^{2}$ de área $(0,50 \times 0,50 \mathrm{~m})$ foi utilizado na coleta, duas amostras ao nível do solo com IL de $95 \%$ e duas com resíduo a $0,15 \mathrm{~m}$. Toda a massa colhida foi acondicionada em sacos plásticos e pesada, de uma subamostra homogênea foi novamente pesada, acondicionada em saco de papel identificado e colocada em estufa de circulação forçada de ar a $55^{\circ} \mathrm{C}$ por 72 horas. Após a secagem, as subamostras foram pesadas novamente para obtenção da massa seca de forragem $\left(\mathrm{Mg} \mathrm{ha}^{-1}\right)$. A massa seca acumulada foi calculada pela diferença de produção entre IL de 95\% e resíduo a $0,15 \mathrm{~m}$, sendo utilizado na extração mineral pelo capim Marandu.

A avaliação química do capim Marandu foi realizada ao final do período experimental aos 350 dias, na condição de manejo adotado. As amostras colhidas foram pesadas e secas em estufa a $55^{\circ} \mathrm{C}$ por 72 horas e moídas em moinho tipo Willey em peneira de $1 \mathrm{~mm}$. Procedeu-se analise de K, Mg, Ca, Cu e Zn (espectrometria de absorção atômica em amostra digerida com solução nitroperclórica); Fósforo total (P) (espectrofotometria UVVis em amostra digerida por solução nitro-perclórica) e Nitrogênio total (N) (método clássico de Kjeldahl). A extração dos nutrientes foi calculada: Extração Anual $\left(\mathrm{kg} \mathrm{ha}^{-1}\right)=$ matéria seca $\left(\mathrm{kg} \mathrm{ha}^{-1}\right) \times$ teor do nutriente $\left(\mathrm{g} \mathrm{kg}^{-1}\right) / 1000$ e Extração Anual por corte $=$ Extração Anual $\left(\mathrm{kg} \mathrm{ha}^{-1}\right) /$ Número de corte.
Os resultados foram submetidos à análise de variância ( $5 \%$ de probabilidade) e análise de regressão aos modelos lineares e quadráticos com uso do software Sisvar (Ferreira, 2011).

\section{RESULTADOS E DISCUSSÃO}

Para os minerais presentes na massa de forragem do capim Marandu com IL de 95\%, houve interação dose de esterco de galinha $\mathrm{x}$ manejo do solo para $\mathrm{Ca}$, $\mathrm{Mg}, \mathrm{N}, \mathrm{Cu}$ e $\mathrm{Zn}$, efeito de dose para $\mathrm{P}$ e sem efeito significativo para o K (Tabela 1).

Houve redução nos teores de Ca e Mg com aumento de dose sem escarificação do solo, talvez em razão da maior frequência e menores intervalos de cortes efetuados com formação de maior número de perfilhos com folhas e colmos mais jovens. Que pode ser explicado possivelmente pelo efeito da diluição (Malavolta et al., 1997). Nessas doses os teores de Ca e Mg estão acima de 3 a $6 \mathrm{~g} \mathrm{~kg}^{-1}$ e 1,5 e 4,0 $\mathrm{g} \mathrm{kg}^{-1}$, respectivamente para Brachiaria brizantha (Werner et al.,1997). Os resultados confirmam Azevedo \& Bonumá (2004) onde coloides inorgânicos e orgânicos adsorvem, eletrostaticamente, esses minerais, retendo-os em forma trocável disponível às plantas. Os resultados corroboram com Silva et al. (2013), que verificaram redução nas concentrações de $\mathrm{Mg}$ com aumento de aplicação de esterco de galinha poedeira (média de 2,87 $\mathrm{g} \mathrm{kg}^{-1}$ ) aos 95 dias após aplicação em capim Marandu.

Para Batista \& Monteiro (2010), o segundo corte na dose de $261 \mathrm{mg} \mathrm{dm}^{-3}$ de $\mathrm{N}$ obteve o teor mais elevado de $\mathrm{Ca}\left(10 \mathrm{~g} \mathrm{~kg}^{-1}\right)$ em lâminas de folhas recém-expandidas do capim Marandu. Barnabé et al. (2007), verificaram aumentos médios de Ca com dejetos líquidos de suínos, com intervalo de corte fixo de 33 dias.

$\mathrm{Na}$ adubação mineral os teores de $\mathrm{Ca}$ e $\mathrm{Mg}$ foram semelhantes às menores doses de esterco e semelhante a dose base. Estes resultados podem ser explicados pelo maior período gasto pelo capim Marandu em atingir 95\% de IL em razão dos menores níveis de $\mathrm{pH}$ e presença desse mineral no solo. De acordo com Eguchi et al. (2016) para a dose zero na profundidade de 0 a 0,10 $\mathrm{m}$, foram observados teores de $1,02 \mathrm{cmol}_{\mathrm{c}} \mathrm{dm}^{-3} \mathrm{de} \mathrm{Ca}$ e de $0,70 \mathrm{cmol}_{\mathrm{c}} \mathrm{dm}^{-3}$ para o Mg. Primavesi et al. (2006) verificaram na massa do capim Marandu a concentração de Ca de 4,0 $\mathrm{g} \mathrm{kg}^{-1}$, com aplicação $100 \mathrm{~kg} \mathrm{ha}^{-1}$ de nitrato de amônio por corte. 
Tabela 1 - Resumo da análise de variância e teste de média para os minerais Ca, $\mathrm{Mg}, \mathrm{K}, \mathrm{N}, \mathrm{P}\left(\mathrm{g} \mathrm{kg}^{-1}\right), \mathrm{Cu}$ e $\mathrm{Zn}\left(\mathrm{mg} \mathrm{kg}^{-1}\right)$, presentes na parte aérea do capim Marandu fertilizado com esterco de galinha e manejo sem e com escarificação do solo

\begin{tabular}{|c|c|c|c|c|c|c|c|c|c|c|c|c|}
\hline & \multicolumn{4}{|c|}{ FV (Quadrados médios) } & \multirow[b]{2}{*}{$\mathrm{CV}(\%)$} & \multirow[b]{2}{*}{ ef } & \multicolumn{5}{|c|}{ Dose $\left(\mathrm{Mg} \mathrm{ha}^{-1}\right)$} & \multirow[b]{2}{*}{ NPK } \\
\hline & $\begin{array}{l}\text { Dose } \\
\text { (D) }\end{array}$ & $\begin{array}{c}\text { Manejo } \\
\text { (M) }\end{array}$ & DXM & $\operatorname{Re}$ & & & 0 & 1,037 & 2,074 & 4,148 & 6,222 & \\
\hline $\mathrm{Ca}$ & 20,44 & 7,61 & $22,66^{*}$ & 3,50 & 14,47 & $\begin{array}{l}\mathrm{s} \\
\mathrm{e}\end{array}$ & $\begin{array}{l}15,45 \mathrm{c} \\
13,55 \mathrm{a}\end{array}$ & $\begin{array}{l}14,95 \mathrm{c} \\
11,27 \mathrm{a}\end{array}$ & $\begin{array}{c}13,93 \mathrm{bc} \\
10,46 \mathrm{a}\end{array}$ & $\begin{array}{c}9,08 \mathrm{a} \\
12,92 \mathrm{a}\end{array}$ & $\begin{array}{c}10,20 a b \\
13,29 a\end{array}$ & $\begin{array}{l}16,40 \mathrm{c} \\
13,75 \mathrm{a}\end{array}$ \\
\hline $\mathrm{Mg}$ & 0,54 & 0,46 & $1,33^{*}$ & 0,43 & 10,37 & $\begin{array}{l}\mathrm{s} \\
\mathrm{e}\end{array}$ & $\begin{array}{l}6,27 \mathrm{ab} \\
5,955 \mathrm{a}\end{array}$ & $\begin{array}{c}6,27 \mathrm{ab} \\
6,12 \mathrm{a}\end{array}$ & $\begin{array}{c}6,32 \mathrm{ab} \\
6,37 \mathrm{a}\end{array}$ & $\begin{array}{l}5,46 a \\
6,90 a\end{array}$ & $\begin{array}{c}5,99 \mathrm{ab} \\
6,89 \mathrm{a}\end{array}$ & $\begin{array}{l}7,20 \mathrm{~b} \\
6,45 \mathrm{a}\end{array}$ \\
\hline K & 7,58 & 31,29 & 3,88 & 3,67 & 17,92 & - & 10,14 & 11,61 & 10,26 & 9,60 & 12,15 & 10,41 \\
\hline $\mathrm{N}$ & 28,15 & 0,25 & $36,96^{*}$ & 8,89 & 19,51 & $\begin{array}{l}\mathrm{s} \\
\mathrm{e}\end{array}$ & $\begin{array}{l}10,62 \mathrm{a} \\
14,62 \mathrm{a}\end{array}$ & $\begin{array}{l}14,62 \mathrm{a} \\
15,33 \mathrm{a}\end{array}$ & $\begin{array}{l}14,65 \mathrm{a} \\
14,98 \mathrm{a}\end{array}$ & $\begin{array}{l}22,30 \mathrm{~b} \\
13,94 \mathrm{a}\end{array}$ & $\begin{array}{l}15,34 a \\
17,79 a\end{array}$ & $\begin{array}{l}14,59 \mathrm{a} \\
14,58 \mathrm{a}\end{array}$ \\
\hline $\mathrm{P}$ & $1,34 *$ & 0,94 & 0,05 & 0,09 & 9,90 & $\mathrm{D}$ & $2,41 \mathrm{a}$ & $3,21 \mathrm{bc}$ & $3,36 \mathrm{c}$ & $3,41 \mathrm{c}$ & $3,38 \mathrm{c}$ & $2,77 b$ \\
\hline $\mathrm{Cu}$ & 206,25 & 57,20 & $95,61 *$ & 3,19 & 10,16 & $\begin{array}{l}\mathrm{s} \\
\mathrm{e}\end{array}$ & $\begin{array}{l}15,12 b \\
14,62 b\end{array}$ & $\begin{array}{c}8,05 \mathrm{a} \\
23,80 \mathrm{c}\end{array}$ & $\begin{array}{l}21,90 \mathrm{c} \\
21,30 \mathrm{c}\end{array}$ & $\begin{array}{l}22,70 \mathrm{c} \\
20,72 \mathrm{c}\end{array}$ & $\begin{array}{l}20,50 \mathrm{c} \\
23,45 \mathrm{c}\end{array}$ & $\begin{array}{c}10,70 \mathrm{a} \\
8,17 \mathrm{a}\end{array}$ \\
\hline $\mathrm{Zn}$ & 23,28 & 46,61 & $150,05^{*}$ & 55,31 & 13,45 & $\begin{array}{l}\mathrm{s} \\
\mathrm{e}\end{array}$ & $\begin{array}{l}59,75 \mathrm{a} \\
51,07 \mathrm{a}\end{array}$ & $\begin{array}{l}52,35 \mathrm{a} \\
56,60 \mathrm{a}\end{array}$ & $\begin{array}{l}57,50 \mathrm{a} \\
59,47 \mathrm{a}\end{array}$ & $\begin{array}{l}51,85 \mathrm{a} \\
58,57 \mathrm{a}\end{array}$ & $\begin{array}{l}61,47 a \\
45,47 a\end{array}$ & $\begin{array}{l}54,75 \mathrm{a} \\
54,65 \mathrm{a}\end{array}$ \\
\hline
\end{tabular}

CV - Coeficiente de variação, Re - Resíduo, NPK - Fonte mineral. * significativo $(\mathrm{P}<0,05)$, média seguida de mesma letra na linha não diferem entre si pelo teste Tukey $(\mathrm{P}>0,05)$. Manejo sem escarificação (s) e com escarificação (e).

No manejo com escarificação para o Ca e Mg, não houve diferença entre as médias em razão da mobilização do solo pela haste do escarificador que proporcionou um aumento de ação por microrganismo na decomposição da matéria orgânica mesmo com baixa dose de esterco aplicado na superfície.

Pela importância do K, com exceção da dose de $6,222 \mathrm{Mg} \mathrm{ha}^{-1}$, os teores estão abaixo da faixa adequada para essa forrageira, que é de 12 a $30 \mathrm{~g} \mathrm{~kg}^{-1}$ (Werner et al., 1997). Os resultados são semelhantes aos de Barnabé et al. (2007) que não verificaram variações significativas na concentração de K com aplicação dejeto liquido de suíno. Silva Neto et al. (2009), com aplicação de resíduo líquido de frigorífico não conseguiram manter as concentrações adequadas de $\mathrm{K}$ no tecido vegetal do capim Marandu. No segundo corte da Brachiaria decumbens, Lana et al. (2010), com uso de doses de $6,25,9,375$ e $12,50 \mathrm{Mg} \mathrm{ha}^{-1}$ de cama de frango, observaram aumentos de 6,1, 8,1 e 7,1 $\mathrm{g} \mathrm{kg}^{-1}$ na concentração de $\mathrm{K}$ em relação ao controle.

Com uso de esterco e manejo sem escarificação, os teores de $\mathrm{N}$ variaram de 14,62 a 22,30 $\mathrm{g} \mathrm{kg}^{-1}$, sendo maiores que Silva Neto et al. (2009) que observaram aumento de 2,80 $\mathrm{g} \mathrm{kg}^{-1}$ na concentração de nitrogênio (N) na biomassa do capim Marandu após receber doses $112,50 \mathrm{~m}^{3} \mathrm{ha}^{-1}$ de resíduo líquido de frigorífico, sendo faixa adequada para essa forrageira de 13 a $20 \mathrm{~g} \mathrm{~kg}^{-1}$
(Werner et al.,1997). No tratamento mineral em ambos os manejos os teores de $\mathrm{N}$ se mostraram semelhantes às doses de esterco, favorecido talvez pelo parcelamento da adubação com N. Os resultados corroboram com Costa et al. (2009b) que observaram no primeiro ano de corte teores de $13,51 \mathrm{~g} \mathrm{~kg}^{-1}$ com dose de $100 \mathrm{~kg} \mathrm{ha}^{-1}$ de N.

Com uso de esterco de galinha, houve aumento nos teores de $\mathrm{P}$, sendo maior que a faixa preconizada por Werner et al. (1997), de 0,8 a 3,0 $\mathrm{g} \mathrm{kg}^{-1}$. Silva et al. (2013), com $15 \mathrm{Mgha}^{-1}$ de esterco de galinha, verificaram $3,0 \mathrm{~g} \mathrm{~kg}^{-1}$, superior aos demais tratamentos. Silva et al. (2012) observaram concentração de $\mathrm{P}$ de 0,32 e 0,21 $\mathrm{mg} \mathrm{dm}{ }^{-3}$ nos tecidos do capim Marandu em períodos de chuva e seca, respectivamente. Lana et al. (2010) observaram no segundo corte concentração $3,12 \mathrm{~g} \mathrm{~kg}$ ${ }^{1}$ de $\mathrm{P}$ com aplicação de $12,50 \mathrm{Mg} \mathrm{ha}^{-1}$ de cama de frango de cama, ou seja, aumento de 1,78 vezes em relação ao controle. No tratamento com adubação mineral o valor de $\mathrm{P}$ se assemelha a menor dose, pelo aumento no intervalo entre cortes ao final e maior produção de massa seca durante todo o período.

Houve interação significativa para os fatores dose de esterco e manejo do solo para os teores de $\mathrm{Cu}$ e de Zn. Para ambos os manejos as concentrações destes minerais estão acima da faixa mínima adequada, de $7 \mathrm{mg} \mathrm{kg}^{-1}$ e $29 \mathrm{mg} \mathrm{kg}^{-1}$, respectivamente (Werner 
et al., 1997). O resultado do tratamento mineral sem escarificação do solo foi semelhante ao tratamento com 1,037 Mg. ha ${ }^{-1}$ de esterco de frango e com escarificação menor que o tratamento zero, em razão da maior produção e de frequências de corte.

Na extração anual, as doses de esterco de galinha provocaram alterações significativas para o $\mathrm{Ca}, \mathrm{Mg}$, $\mathrm{K}, \mathrm{P}$ e Zn e para os demais minerais ocorreu interação dose de esterco e manejo do solo (Tabela 2). Para o $\mathrm{N}$ e Cu, sem e com manejo de escarificação do solo, as maiores extrações ocorreram com maiores doses de esterco de galinha e com tratamento mineral, em razão da maior produção de massa seca com IL de 95\%, mesmo com efeito climático principalmente de baixa temperatura nesta região, (Figura 1). Devido a maior produção de massa seca no tratamento mineral, os resultados foram maiores que Costa et al. (2009a), com três cortes realizados ao ano e por Primavesi et al. (2006) com nitrato de amônio após quatro cortes consecutivos na estação chuvosa.

Com as maiores doses de esterco de galinha $\mathrm{x}$ manejo do solo o $\mathrm{N}$ foi o mineral de maior extração, mas com efeito de dose houve maior extração anual de $\mathrm{K}, \mathrm{Ca}$, $\mathrm{Mg}, \mathrm{P}$ e $\mathrm{Zn}$ e com tratamento mineral foram observadas maiores extrações de $\mathrm{Ca}, \mathrm{K}, \mathrm{Mg}, \mathrm{P}$ e $\mathrm{Zn}$, o Ca pode ser devido ao maior período entre cortes com efeito da diluição. Primavesi et al. (2006) com uso de duas fontes de nitrogênio e em todos os tratamentos, as extrações dos macronutrientes foram maiores para $\mathrm{K}$ seguidas de $\mathrm{N}, \mathrm{Ca}, \mathrm{Mg}, \mathrm{P}$ e $\mathrm{S}$ e dos micronutrientes na seguinte ordem decrescente: Fe, Mn, Zn e Cu. E na extração anual por corte houve efeito significativo para interação dose e manejo para $\mathrm{Ca}$ e $\mathrm{Cu}$, efeito de dose para $\mathrm{P}$ e demais mineral sem variações mesmo com aumento de número de cortes efetuados nas maiores doses, mas certamente os resultados contribuem para o planejamento do número pastejo com animais para reposição desses minerais ao solo.

A extração de $\mathrm{Mg}, \mathrm{K}$ e $\mathrm{P}$ foi linear com efeito de doses de esterco e para $\mathrm{N}$ foi linear com interação entre dose de esterco x manejo (Figura 2) e somente para o Ca não houve ajuste aos dois modelos propostos. Os ajustes lineares com incremento de minerais extraídos

Tabela 2 - Resumo da análise de variância e teste de média para extração $\left(\mathrm{kg} \mathrm{kg}^{-1}\right)$ e extração por número de corte $\left(\mathrm{kg} \mathrm{kg}^{-1} \mathrm{NC}^{-1}\right)$, pela parte aérea do capim Marandu fertilizado com esterco de galinha e manejo sem e com escarificação do solo

\begin{tabular}{|c|c|c|c|c|c|c|c|c|c|c|c|c|}
\hline & \multicolumn{4}{|c|}{ FV (Quadrados médios) } & \multirow{2}{*}{$\mathrm{CV}(\%)$} & \multirow{2}{*}{ ef } & \multicolumn{5}{|c|}{ Dose $\left(\mathrm{Mg} \mathrm{ha}^{-1}\right)$} & \multirow{2}{*}{ NPK } \\
\hline & Dose(D) & Manejo(M) & DXM & $\operatorname{Re}$ & & & 0 & 1,037 & 2,074 & 4,148 & 6,222 & \\
\hline \multicolumn{13}{|c|}{ Extração anual (kg.ha-1 $)$} \\
\hline $\mathrm{Ca}$ & $25070,64 *$ & 89,56 & 5422,12 & 2268,29 & 23,03 & $\mathrm{D}$ & $147,32 \mathrm{a}$ & $173,45 \mathrm{ab}$ & $192,10 \mathrm{ab}$ & $194,90 \mathrm{ab}$ & $224,21 b$ & $308,58 \mathrm{c}$ \\
\hline $\mathrm{Mg}$ & $6142,86^{*}$ & 479,07 & 764,23 & 503,49 & 21,69 & $\mathrm{D}$ & $62,38 \mathrm{a}$ & $83,56 \mathrm{ab}$ & $101,97 \mathrm{bc}$ & $109,80 \mathrm{cbd}$ & $123,29 \mathrm{~cd}$ & $139,80 \mathrm{~d}$ \\
\hline $\mathrm{K}$ & $16714,97 *$ & 11819,93 & 2357,04 & 2771,73 & 29,96 & $\mathrm{D}$ & $105,16 \mathrm{a}$ & $157,50 \mathrm{ab}$ & $169,99 \mathrm{ab}$ & $171,81 \mathrm{ab}$ & $236,16 b$ & $213,61 b$ \\
\hline \multirow[t]{2}{*}{$\mathrm{N}$} & 47860,41 & 61,45 & $17820,94 *$ & 6209,42 & 30,98 & s & $104,77 \mathrm{a}$ & $194,90 \mathrm{ab}$ & $200,65 \mathrm{ab}$ & $422,15 \mathrm{c}$ & $297,72 b c$ & $312,76 \mathrm{bc}$ \\
\hline & & & & & & $\mathrm{e}$ & $159,47 \mathrm{a}$ & $219,42 \mathrm{ab}$ & $269,36 \mathrm{ab}$ & $239,21 \mathrm{ab}$ & $344,30 b$ & $287,62 \mathrm{ab}$ \\
\hline $\mathrm{P}$ & $1727,77 *$ & 512,85 & 72,25 & 105,20 & 20,17 & $\mathrm{D}$ & $25,01 \mathrm{a}$ & $42,97 b$ & $54,39 \mathrm{bc}$ & $60,75 \mathrm{c}$ & $65,11 \mathrm{c}$ & $56,84 b c$ \\
\hline \multirow[t]{2}{*}{$\mathrm{Cu}$} & 0,10 & 0,01 & $0,02 *$ & 0,004 & 24,29 & $\mathrm{~s}$ & $0,10 \mathrm{a}$ & $0,15 \mathrm{a}$ & $0,30 \mathrm{bc}$ & $0,42 \mathrm{c}$ & $0,40 \mathrm{c}$ & $0,23 \mathrm{ab}$ \\
\hline & & & & & & $\mathrm{e}$ & $0,15 \mathrm{a}$ & $0,33 b$ & $0,38 b$ & $0,35 b$ & $0,45 b$ & $0,15 \mathrm{a}$ \\
\hline \multirow[t]{2}{*}{$\mathrm{Zn}$} & $0,32 *$ & 0,0001 & 0,07 & 0,030 & 19,49 & $\mathrm{D}$ & $0,56 a$ & $0,73 \mathrm{ab}$ & $0,93 \mathrm{bc}$ & $0,98 \mathrm{bc}$ & $1,02 \mathrm{c}$ & $1,10 \mathrm{c}$ \\
\hline & \multicolumn{12}{|c|}{ Extração anual por corte $\left(\mathrm{kg} \cdot \mathrm{ha}^{-1} \cdot \mathrm{NC}^{-1}\right)$} \\
\hline $\mathrm{Ca}$ & 390,07 & 58,11 & $376,57 *$ & 122,49 & 27,08 & $\mathrm{e}$ & $46,44 \mathrm{ab}$ & $47,86 \mathrm{ab}$ & $50,04 \mathrm{~b}$ & $26,08 \mathrm{a}$ & $25,29 \mathrm{a}$ & $56,12 b$ \\
\hline $\mathrm{Mg}$ & 43,59 & 11,39 & 35,10 & 24,26 & 24,62 & - & $17,93 a$ & $19,68 \mathrm{a}$ & $22,28 \mathrm{a}$ & $19,22 \mathrm{a}$ & $17,58 \mathrm{a}$ & $23,35 \mathrm{a}$ \\
\hline $\mathrm{K}$ & 91,92 & 386,47 & 40,13 & 100,27 & 29,92 & - & $29,34 \mathrm{a}$ & $36,59 \mathrm{a}$ & $36,73 a$ & $29,42 \mathrm{a}$ & $33,28 \mathrm{a}$ & $35,38 \mathrm{a}$ \\
\hline $\mathrm{N}$ & 313,97 & 12,20 & 269,46 & 165,70 & 26,98 & - & $36,88 \mathrm{a}$ & $47,99 a$ & $50,25 \mathrm{a}$ & $55,61 \mathrm{a}$ & $45,50 \mathrm{a}$ & $49,96 a$ \\
\hline$P$ & $20,46^{*}$ & 15,36 & 3,81 & 6,16 & 25,52 & $\mathrm{D}$ & $7,11 \mathrm{a}$ & $10,13 \mathrm{ab}$ & $11,93 \mathrm{~b}$ & $10,51 \mathrm{ab}$ & $9,21 \mathrm{ab}$ & $9,49 \mathrm{ab}$ \\
\hline \multirow[t]{2}{*}{$\mathrm{Cu}$} & 0,002 & 0,0006 & 0,001 & $0,0002 *$ & 25,65 & $\mathrm{~s}$ & $0,04 \mathrm{ab}$ & $0,02 \mathrm{ab}$ & $0,08 \mathrm{c}$ & $0,06 \mathrm{bc}$ & $0,05 \mathrm{ab}$ & $0,03 \mathrm{ab}$ \\
\hline & & & & & & e & $0,04 \mathrm{ab}$ & $0,07 \mathrm{c}$ & $0,07 \mathrm{bc}$ & $0,06 \mathrm{bc}$ & $0,06 b c$ & $0,02 \mathrm{a}$ \\
\hline $\mathrm{Zn}$ & 0,003 & 0,000004 & 0,001 & 0,001 & 24,72 & - & $0,16 a$ & $0,17 \mathrm{a}$ & $0,20 \mathrm{a}$ & $0,17 \mathrm{a}$ & $0,14 \mathrm{a}$ & $0,18 \mathrm{a}$ \\
\hline
\end{tabular}

CV - Coeficiente de variação, Re - Resíduo, NPK - Fonte mineral. * significativo $(\mathrm{P}<0,05)$, média seguida de mesma letra na linha não diferem entre si pelo teste Tukey $(\mathrm{P}>0,05)$. Manejo sem escarificação (s) e com escarificação (e). 
(A)

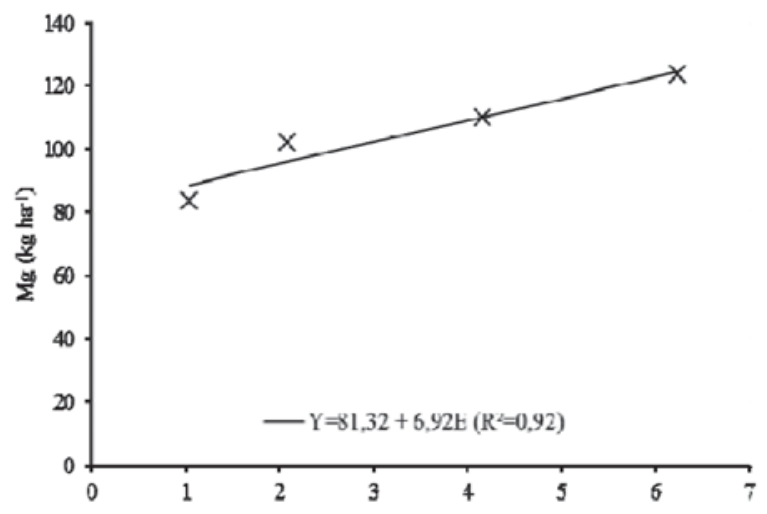

(C)

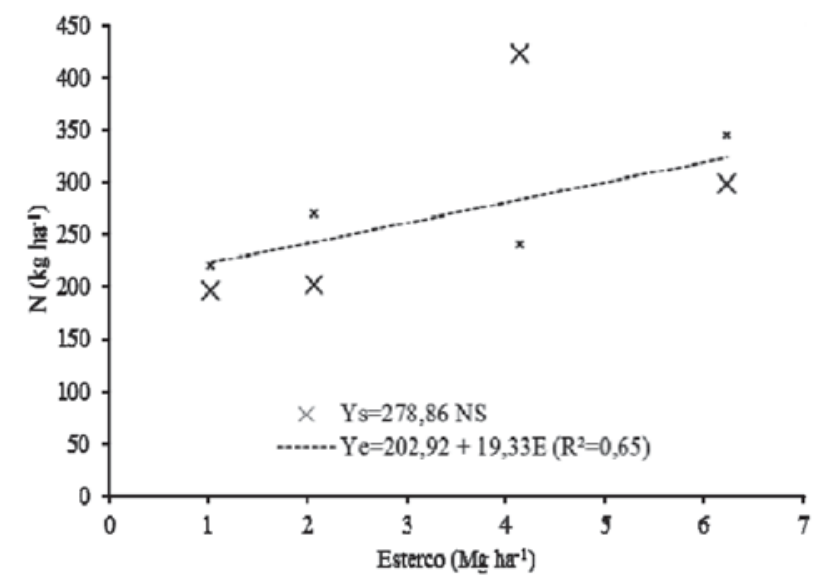

(E)

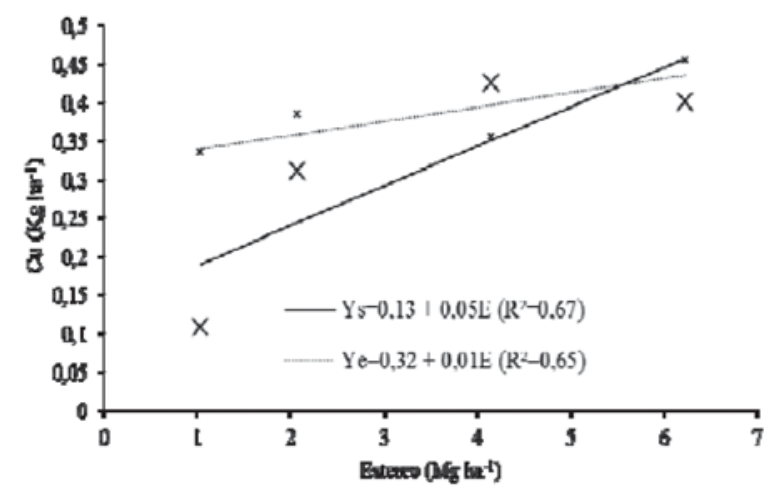

(B)

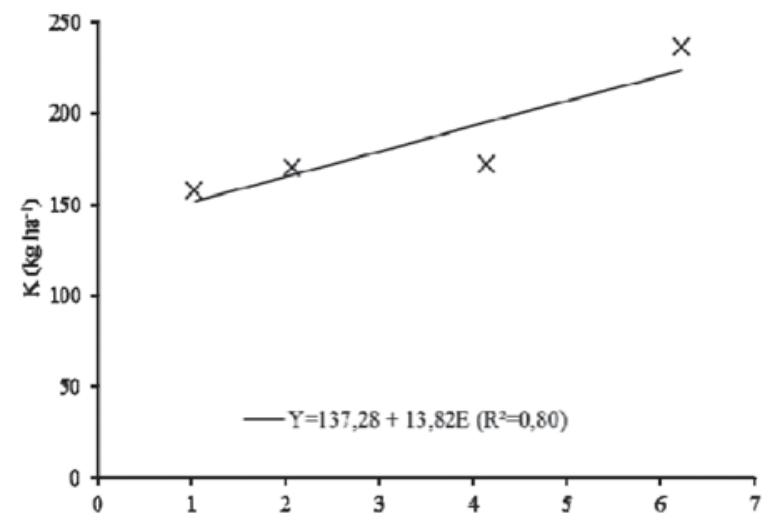

(D)

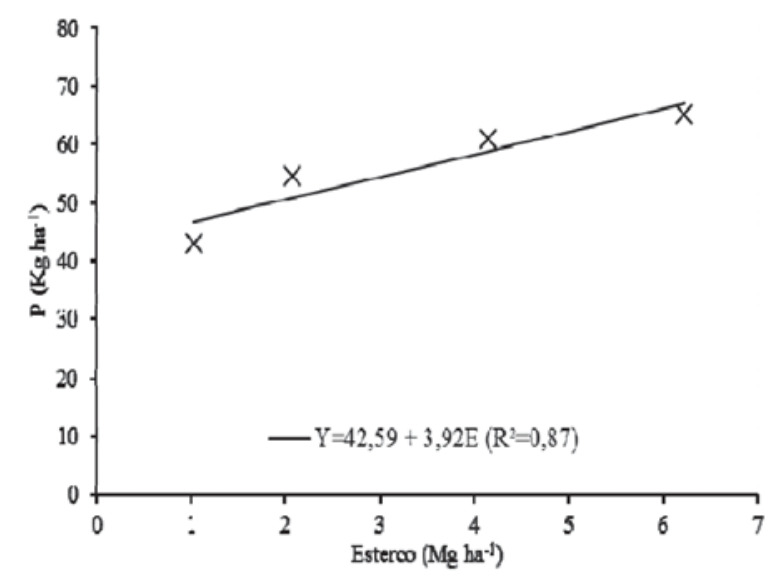

(F)

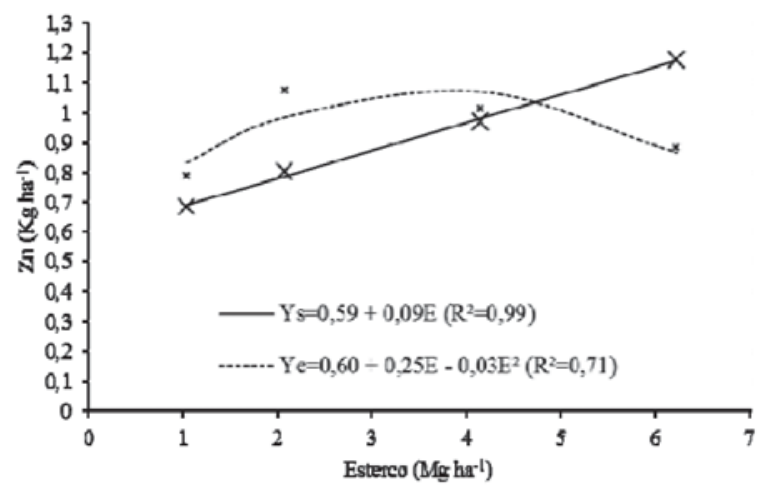

Figura 2 - Extração mineral pela massa de forragem do capim Marandu fertilizado com esterco de galinha e manejo sem e com escarificação do solo (s,e). 
pela massa de forragem do capim Marandu foram motivados pelo aumento na produção de forragem acumulada devido ao número de cortes efetuados com aumento de fertilização de esterco de galinha.

$\mathrm{Na}$ extração de Mg entre as doses de 1,037 e 6,222 $\mathrm{Mg} \mathrm{ha}^{-1}$ de esterco de galinha houve aumento de 39,72 $\mathrm{kg} \mathrm{ha}^{-1}$ devido a massa seca acumulada (MS) de $5,91 \mathrm{Mg}$ ha $^{-1}$ durante os 350 dias (Figura 2A). Os resultados confirmam Costa et al. (2009a), que observaram no ajuste linear um aumento de quatro vezes em relação à testemunha, com o acréscimo de doses de nitrogênio nas extrações de magnésio pelo capim Marandu. Primavesi et al. (2006) constataram ajuste quadrático com aumento de 5,4 vezes na extração de $\mathrm{Mg}$ na dose de $400 \mathrm{~kg} \mathrm{ha}^{-1}$ ano ${ }^{1}$ de $\mathrm{N}$ aplicado, em relação à testemunha.

O aumento da extração do K pelo capim Marandu em relação à menor dose de esterco de galinha foi de $7,92,9,08$ e $49,94 \%$ para as doses de 2,074, 4,148 e $6,222 \mathrm{Mg} \mathrm{ha}^{-1}$, respectivamente. Os resultados de certa forma, corroboram com Costa et al. (2009a), que verificaram extração próximas de $\mathrm{K}$ de $251,87 \mathrm{~kg} \mathrm{ha}^{-1}$ no segundo ano de avaliação de capim Marandu e de Primavesi et al. (2006) que relataram extração de $429 \mathrm{~kg} \mathrm{ha}^{-1}$, sendo ambos com maior adubação e produção de massa seca.

$\mathrm{Na}$ interação dose de esterco $\mathrm{x}$ manejo com escarificação, a extração de $\mathrm{N}$ com dose de $6,222 \mathrm{Mg}$ $\mathrm{ha}^{-1}$ foi de $344,30 \mathrm{~kg} \mathrm{ha}^{-1}$, ou seja, aumento de $56,91 \%$ em relação dose de 1,037 $\mathrm{Mg} \mathrm{ha}^{-1}$ de esterco (Figura 2C). A escarificação do solo manteve constante a concentração de N no capim Marandu mesmo com aumento de dose de esterco. Segundo Azeez e Averbeke (2010), aos 120 dias $80 \%$ do $\mathrm{N}$ presente no esterco de galinha é mineralizada, com dependência continua de ação e morte de microrganismos. Primavesi et al. (2006) verificaram que a extração de nitrogênio foi linear para duas fontes (ureia e o nitrato de amônio) e entre doses de 200 e $800 \mathrm{~kg} / \mathrm{ha} /$ ano de N, a extração foi 2,5 e 2,86 vezes maior, respectivamente. Com fertilização de esterco de galinha os valores verificados de extração de N são maiores do que K. Relacionado também, pelo sistema de manejo adotado com IL de $95 \%$ com maiores números de cortes e produção acumulada de massa de forragem do capim Marandu. Os resultados diferem de Primavesi et al. (2006) e Costa et al. (2009a), que verificaram extração maior de $\mathrm{K}$ em relação ao $\mathrm{N}$ pelo capim Marandu.
$\mathrm{O}$ aumento de extração de $\mathrm{P}$ entre as doses de esterco aplicado foi de 22,14 $\mathrm{kg} \mathrm{ha}^{-1}$. Costa et al. (2009a) observaram no modelo quadrático o aumento na extração de $\mathrm{P}$ de $6,26 \mathrm{~kg} \mathrm{ha}^{-1}$ no primeiro ano de avaliação entre doses de $100 \mathrm{e} 300 \mathrm{~kg} \mathrm{ha}^{-1} \mathrm{de}$ N. Os resultados corroboram com Primavesi et al. (2006) que verificaram entre mesmas doses de $\mathrm{N}$ aumento de extração de 13 e $23 \mathrm{~kg} \mathrm{ha}^{-1}$ para ureia e nitrato de amônio, respectivamente.

$\mathrm{Na}$ interação entre doses de esterco e manejo do solo houve ajuste linear para o $\mathrm{Cu}$ (Figura 2E). Comparando a extração desse micronutriente na dose $6,222 \mathrm{Mg} \mathrm{ha}^{-1}$ em relação à dose de $1,037 \mathrm{Mg} \mathrm{ha}^{-1} \mathrm{de}$ esterco, o aumento foi de 3,72 e 1,35 vezes, sem e com escarificação do solo, respectivamente. A maior variação de extração com aplicação de esterco de galinha sem a escarificação do solo é por causa do aumento na massa seca acumulada (MS) pelo capim Marandu nas maiores doses. Braz et al. (2004) encontraram extração de $\mathrm{Cu}$ de $0,69 \mathrm{~kg} \mathrm{ha}^{-1}$ nas folhas do capim Marandu aos 105 dias.

$\mathrm{Na}$ interação dose de esterco e manejo sem escarificação do solo houve ajuste linear para o $\mathrm{Zn}$ com aumento de $0,49 \mathrm{~kg} \mathrm{ha}^{-1}$ entre doses de esterco aplicado (Figura 2F). E na interação entre dose de esterco e manejo com escarificação do solo o ajuste foi quadrático com extração máxima de $1,06 \mathrm{~kg} \mathrm{ha}^{-1}$ com dose de 2,77 $\mathrm{Mg} \mathrm{ha}^{-1}$ de esterco. Braz et al. (2004) observaram valor máximo de extração de $\mathrm{Zn}$ de $0,204 \mathrm{~kg} \mathrm{ha}^{-1}$ nas folhas de capim Marandu. A extração de $\mathrm{Zn}$ foi maior que Cu no capim Marandu, estando de acordo com Primavesi et al. (2006) e Costa et al. (2009a).

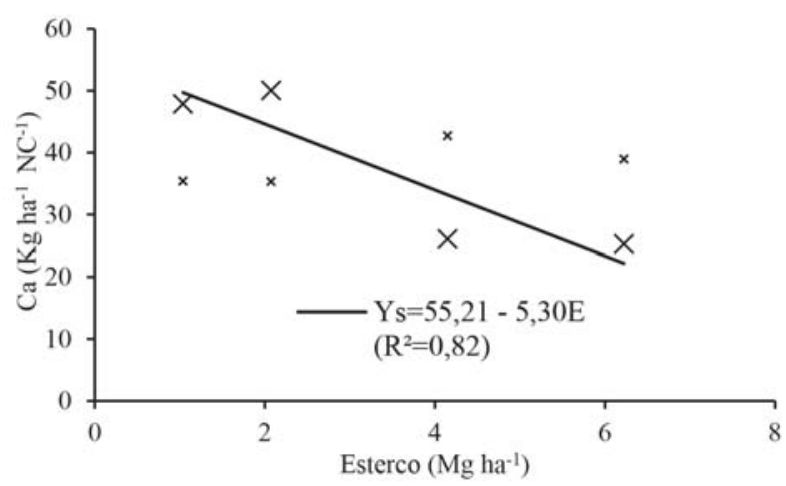

Figura 3 - Extração mineral por corte no capim Marandu fertilizado com esterco de galinha e manejo sem e com escarificação do solo $(\mathrm{s}, \mathrm{e})$. 
$\mathrm{Na}$ interação dose de esterco e sem o manejo de escarificação do solo para extração mineral de Ca por corte efetuado no capim Marandu houve ajuste ao modelo linear decrescente entre as doses de esterco $(1,037 \mathrm{e}$ $6,222 \mathrm{Mg} \mathrm{ha}^{-1}$ ), o que demonstra nas maiores doses de esterco de galinha o efeito da diluição em razão do maior número perfilhos, maior quantidade de folhas jovens em razão dos cortes efetuados ao longo do ano.

\section{CONCLUSÃO}

$\mathrm{ON}$ foi o mineral mais extraído pela massa de forragem do capim Marandu com uso de esterco de galinha e manejo mecânico do solo.

Em razão do aumento da dose de esterco de galinha e da produção de massa de forragem do capim Marandu houve aumentou na extração de $\mathrm{K}, \mathrm{Ca}, \mathrm{Mg}, \mathrm{P}, \mathrm{Zn}$ e Cu .

\section{LITERATURACITADA}

ASHJAEI, S.; MILLER, W.P.; CABRERA, M.L. et al. M. Arsenic in Soils and Forages from Poultry LitterAmended Pastures. International Journal of Environmental Research and Public Health, v.8, p.1534-1546, 2011.

AZEEZ, J.O.; AVERBEKE, V.W. Nitrogen mineralization potential of three animal manures applied on a Sandy Clay loam soil. Bioresource Technology, v.101, n.14, p.5645-5651, 2010.

AZEVEDO, A.C. de; BONUMÁ, A.S. Partículas coloidais, dispersão e agregação em latossolos. Ciência Rural, Santa Maria, v.34, n.2, p.609-617, 2004.

BARNABÉ, M.C.; BENEVAL, R.; LOPES, E.L. et al. Produção e composição químico-bromatológica da Brachiaria Brizantha cv. marandu adubada com dejetos líquidos de suínos. Ciência Animal

Brasileira, Goiânia, v.8, n.3, p.435-446, 2007.

BATISTA, K.; MONTEIRO, F.A. Variações nos teores de potássio, cálcio e magnésio em capim-marandu adubado com doses de nitrogênio e de enxofre.

Revista Brasileira de Ciência do Solo, Viçosa, v.34, n.1, p.151-161, 2010.

BRASIL. Ministério da Agricultura, Pecuária e Abastecimento. Instrução Normativa n. 25, de 23 de julho de 2009. Diário Oficial da República Federativa do Brasil, Brasília, DF, 28 de julho de 2009. Seção 1, p.20, 2009.
BRAZ, A.J.B.P.; SILVEIRA, P.M. da; KLIEMANN, H.J. et al. Acumulação de nutrientes em folhas de milheto e dos capins braquiária e mombaça.

Pesquisa Agropecuária Tropical, Goiânia, v.34, n.2, p.83-87, 2004.

COSTA, K.A. de P.; FAQUIN, V.; OLIVEIRA, I.P. et al. Extração de nutrientes do capim-marandu sob doses e fontes de nitrogênio. Revista

Brasileira de Saúde e Produção Animal, Salvador, v.10, n.4, p.801-812, 2009 (a).

COSTA, K.A. de P.; FAQUIN, V.; OLIVEIRA, I.P. et al. Doses e fontes de nitrogênio na nutrição mineral do capim-marandu. Ciência Animal Brasileira, Goiânia, v.10, n.1, p.115-123, 2009 (b).

DIFANTE, G. dos S.; EUCLIDES, V.P.B.; NASCIMENTO JUNIOR, D. et al., Ingestive behaviour, herbage intake and grazing efficiency of beef cattle steers on Tanzania guineagrass subjected to rotational stocking managements. Revista Brasileira de Zootecnia, v.38, p.1001 1008, 2009.

EGUCHI, E.S.; CECATO, U.; MUNIZ, A.S. et al. Physical and chemical changes in soil fertilized with poultry manure with and without chiseling. Revista Brasileira de Engenharia Agrícola e Ambiental, v.20, n.4, p.316-321, 2016.

FERREIRA, D.F. Sisvar: a computer statistical analysis system. Ciência e Agrotecnologia, Lavras, v.35, n.6, p.1039-1042, 2011.

LANA, R.M.Q.; ASSIS, D.F. de; SILVA, A. de A. et al. Alterações na produtividade e composição nutricional de uma pastagem após segundo ano de aplicação de diferentes doses de cama de frango. Bioscience Journal, Uberlândia, v.26, n.2, p.249-256, 2010.

MALAVOLTA, E.; VITTI G.C.; OLIVEIRA S.A. Avaliação do estado nutricional das plantas. $2^{\mathrm{a}}$ ed. Piracicaba, Potafós. 1997. 319p.

MENEZES, J.F.S.; ALVARENGA, R.C.; SILVA, G.P. et al. Cama-de-frango na agricultura: perspectivas e viabilidade técnica e econômica. Boletim Técnico, 3. Rio Verde, GO: Fundação do Ensino Superior de Rio Verde, 2004. 28 p. 
OLIVEIRA, E.L. de. Sugestão de adubação e calagem para culturas de interesse econômico no Estado do Paraná.

Londrina: IAPAR, 2003. 30p.

PEDREIRA, B.C.; PEDREIRA, C.G.S.; SILVA, S.C. Acúmulo de forragem durante a rebrotação de capim-xaraés submetido a três estratégias de desfolhação. Revista Brasileira de

Zootecnia, Viçosa, v.38, n.4, p.618-625, 2009.

PRIMAVESI, A.C.; PRIMAVESI, O.; CORRÊA, L.A. et al. Nutrientes na fitomassa de capim-Marandu em função de fontes e doses de nitrogênio.

Ciência e Agrotecnologia, v.30, n.3, p.562$568,2006$.

SILVA NETO, S.P. da; SILVA, J.E.C. da; SANTOS, A.C. dos. et al. Características agronômicas e nutricionais do capim-Marandu em função da aplicação de resíduo líquido de frigorífico. Acta Scientiarum. Animal Sciences, Maringá, v.32, n.1, p.9-17. 2009.

SILVA, A.A.; SIMIONI, G.F.; LUCENA, A. Efeito da adubação orgânica no crescimento do capim Brachiaria Brizantha cv. Marandu em Parecis/ Rondônia. Enciclopédia Biosfera, Centro Científico Conhecer, Goiânia, v.9, n.16, p.923-932, 2013.

Recebido para publicação em 5/4/2017 e aprovado em 4/9/2017
SILVA, C.C.; SANTOS, A.C. dos; SILVA, G.F. et al. Resposta do capim Marandu (Brachiaria brizantha Stapf) a aplicação de NPK e fontes de matéria orgânica. Amazônia: Ciência \& Desenvolvimento, Belém, v.7, p.43-57, 2012.

TRINDADE, J.K. da; DA SILVA, S.C.; SOUZA JÚNIOR, S.J. et al. Composição morfológica da forragem consumida por bovinos de corte durante o rebaixamento do capim marandu submetido a estratégias de pastejo rotativo. Pesquisa Agropecuária Brasileira, v.42, p.883 890, 2007.

VOLTOLINI, T.V.; SANTOS, F.A.P.; MARTINEZ, J.C. et al. Características produtivas e qualitativas do capim-elefante pastejado em intervalo fixo ou variável de acordo com a interceptação da radiação fotossinteticamente ativa. Revista Brasileira de Zootecnia, Viçosa, v.39, n.5, p.1002-1010, 2010.

WERNER, J.C.; PAULINO, V.T.; CANTARELLA, H. et al. Forrageiras. In: RAIJ, B. VAN;

CANTARELLA, H.; QUAGGIO, J.A.; FURLANI, A.M.C. Recomendações de adubação e calagem para o Estado de São Paulo. 2.ed. Campinas: Instituto Agronômico de Campinas, 1997. p.263-273. 